\title{
Time-of-Flight Effects in Microwave Transmission Through a Thin Metal Slab
}

\author{
Henningsen, J. O.; Falk, David S.
}

Published in:

Physical Review Letters

Link to article, DOI:

10.1103/PhysRevLett.26.1174

Publication date:

1971

Document Version

Publisher's PDF, also known as Version of record

Link back to DTU Orbit

Citation (APA):

Henningsen, J. O., \& Falk, D. S. (1971). Time-of-Flight Effects in Microwave Transmission Through a Thin Metal Slab. Physical Review Letters, 26(19), 1174-1177. https://doi.org/10.1103/PhysRevLett.26.1174

\section{General rights}

Copyright and moral rights for the publications made accessible in the public portal are retained by the authors and/or other copyright owners and it is a condition of accessing publications that users recognise and abide by the legal requirements associated with these rights.

- Users may download and print one copy of any publication from the public portal for the purpose of private study or research.

- You may not further distribute the material or use it for any profit-making activity or commercial gain

- You may freely distribute the URL identifying the publication in the public portal

If you believe that this document breaches copyright please contact us providing details, and we will remove access to the work immediately and investigate your claim. 
Time-of-Flight Effects in Microwave Transmission Through a Thin Metal Slab

\author{
J. O. Henningsen* \\ Physics Laboratory I, The Technical University of Denmark, Lyngby, Denmark
}

and

David S. Falk

Department of Physics, University of Maryland, College Park, Maryland 20742, and Physics Laboratory I, The Technical University of Denmark, Lyngby, Denmark

(Received 15 January 1971)

\begin{abstract}
A 45-GHz size-effect experiment on Ag revealed significant structure in the combined transmission and leakage signal below the first-belly-electron size effects. The oscillations, which behave differently for different field orientations, are shown to be due to the time-of-flight phase delay of electron trajectories across the sample. The signal is calculated assuming circular orbits and using parameters from the literature. We suggest a modified experiment for obtaining Fermi velocities.
\end{abstract}

In a size effect experiment ${ }^{1}$ on silver at microwave frequencies, where the transmitted signal is allowed to interfere with a leak signal, we have observed a significant amount of structure below the first-order size effect of the belly orbits. With the magnetic field along the [100] direction, the first-order line has an oscillatory tail which extends down to $B / B_{\text {S.E. }} \approx 0.7, B_{\text {S.E. }}$ being the cyclotron-resonance cutoff field (Fig. 1). For $\vec{B}$ parallel to the [011] direction (Fig. 2), there is an oscillatory signal, extending almost up to the size-effect line, with a maximum amplitude near $B / B_{\text {S.E. }} \approx 0.5$.

We interpret these results as being caused by belly electrons transferring microwave energy across the Ag slab in their ballistic motion. For $\vec{B} \|[100]$ the signal is caused mainly by electrons which collide with both surfaces (type-II trajectories, Fig. 3), whereas for $\vec{B} \|[011]$ the signal is primarily due to electrons which skim along

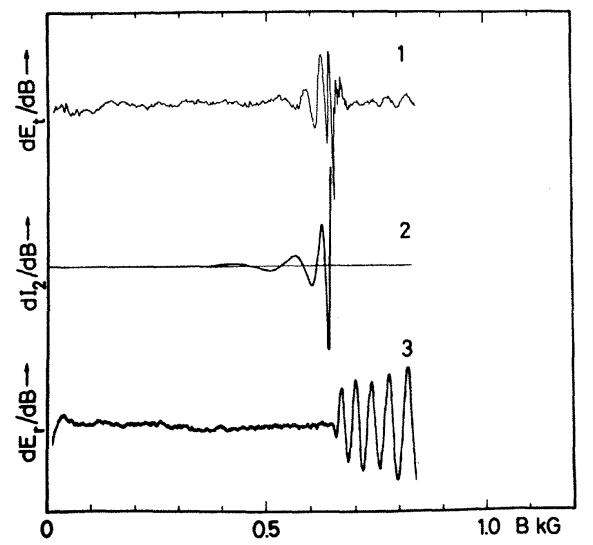

FIG. 1. Curve 1, recorded transmission spectrum for $\vec{B} \|$ [100]; curve 2, calculated transmission spectrum as given by type- $\Pi$ orbits; and curve 3 , recorded reflection spectrum. one surface and collide with the other (type-I trajectories). The electrons arrive at the second surface with a phase delay determined essentially by their time of transit across the sample. This phase, modulo $2 \pi$, is measured by the interference of the transmitted signal with the leakage signal. Since the time of transit is a function of the magnetic field, the interference product oscillates with $B$. One period of this oscillation thus corresponds to a change of one microwave period in the time of transit of the electrons. For a general Fermi surface, the field at the second surface is a sum of contributions from all electrons connecting the two surfaces. The structure in the transmitted signal is then determined by those electrons (if any) arriving with extremal phase delay. ${ }^{2}$

The experiment was performed at a frequency of $44.8 \mathrm{GHz}$ in a two-cavity transmission spectrometer. The $0.235-\mathrm{mm}$ sample (resistivity

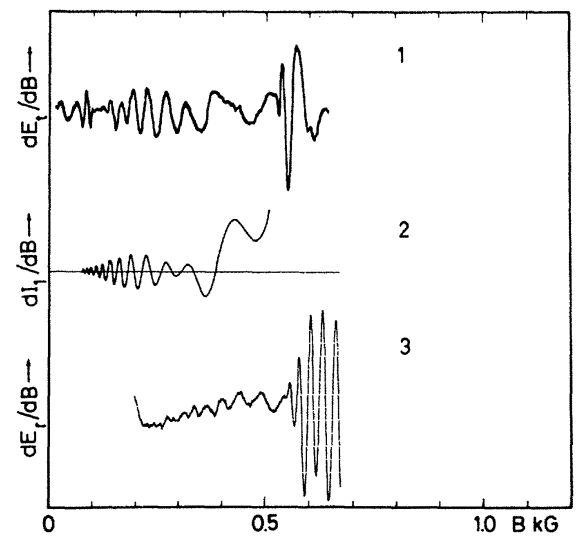

FIG. 2. Curve 1, recorded transmission spectrum $\overrightarrow{\mathrm{B}} \|$ [011]; curve 2, calculated transmission spectrum as given by type-I orbits; and curve 3 , recorded reflection spectrum. 
(a)

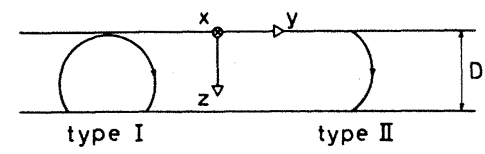

(b)

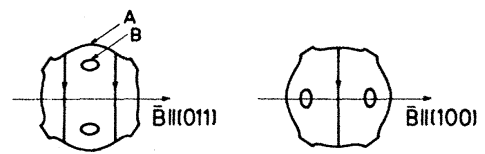

FIG. 3. (a) The two types of orbits considered in the text, and (b) the location on the Fermi surface of the stationary phase orbits.

ratio 7500 ) was attached to one cavity by a thin layer of paraffin. The other side of the sample was covered by 1-mil Mylar foil and lightly pressed against the second cavity. This arrangement allowed some microwave power to leak around the sample, the isolation being approximately $60 \mathrm{~dB}$. Using magnetic field modulation at $270 \mathrm{~Hz}$ and subsequent phase-sensitive detection, we measured the magnetic field derivative of the combined transmission and leakage signal. By recording the reflection spectra also, we determined the cyclotron-resonance cutoff fields. This information aided in the interpretation of the transmission results.

The detected signal is determined by the weighted average of the current over the skin depth at the second surface. The electric field, of frequency $\omega$, is perpendicular to $\vec{B}$ (both fields are parallel to the sample surface) and is asssumed to have an exponential spatial dependence with complex skin depth $\delta$. Using the Chambers expression for the current, ${ }^{3}$ we have computed the detected signal for the experimental conditions

$$
\begin{aligned}
& \delta / R_{c} \ll 1, \omega_{c} \tau ; \quad \delta / D \ll 1 ; \omega \delta / \omega_{c} R_{c} \ll 1 ; \\
& \omega / \omega_{c} \gg 1 ;
\end{aligned}
$$

where $R_{c}$ is the cyclotron radius of the relevant electrons, $\omega_{c}$ their cyclotron frequency, and $D$ the sample thickness. We further assumed that the phase of the leak signal was equal to that of the incident signal, that the orbits were circular (those of the relevant electrons in Ag differ from circles by only a few percent ${ }^{4}$ ), and that there was diffuse reflection at the sample surfaces. Retardation effects ${ }^{5}$ were included.

The number of type-I electrons is proportional to $\delta / R_{c}$, and the time they spend in the skin layer to $\left(\delta / R_{c}\right)^{1 / 2}$; so their contribution is proportional to $\left(\delta / R_{c}\right)^{3 / 2}$. The type-II electrons spend a smaller time, proportional to $\delta / R_{c}$, in the skin layer. However, the number of type-II electrons that contribute effectively to the current is restricted only by the requirement that they arrive at the second surface with a phase spread less than $\pi$. This makes their number proportional to $\left(\omega_{c} / \omega\right)^{1 / 2}$ and consequently, by (1), much greater than the number of type-I electrons. Their contribution is thus proportional to $\delta \omega_{c}{ }^{1 / 2} / R_{c} \omega^{1 / 2}$.

In comparing with experiment, we neglected the rosette orbits $(\vec{B} \|[100])$ and the dog's-bone orbits $(\vec{B} \|[011])$. They give rise to cyclotronresonance signals which are smaller by orders of magnitude than those of the belly electrons. Furthermore, they cause no observable effect in the relevant range of magnetic field. The open orbits present in the [011] direction were neglected because of their small number. Because of their shape they cannot, in any event, contribute to type-I oscillations.

For $\vec{B}$ along the [100] direction, we have approximated the Fermi surface by a cylinder. The various parameters were chosen as follows. For $m_{c} / m_{0}$, the ratio of the cyclotron to the bare mass, we used the value 0.95 determined from the reflection spectrum. The radius of the cylindrical Fermi surface $k_{\perp}$ was taken to $\mathrm{be}^{6,7} 1.16$ $\times 10^{10} \mathrm{~m}^{-1}$ and the sample thickness, $D=0.235$ $\mathrm{mm}$, was determined by $k_{\perp}$ together with the measured spacing of the equidistant set of size effects. For the skin depth we used $\delta=\delta_{0} e^{i x}$, where $\delta_{0}$ as well as $\chi$ were assumed to be independent of the magnetic field. We used $\delta_{0}=1.20 \times 10^{-7} \mathrm{~m}^{8}$ and $\chi=\pi / 6 .^{9}$ Finally, the value $\omega \tau \approx 30$ was estimated from the cyclotron-resonance spectrum. For these parameters, the type-II trajectories dominate. The magnetic field derivative of the averaged current is compared with the experimental tracing in Fig. 1. Note that this represents a zero-parameter fit since no ad hoc adjustments were introduced. The agreement is satisfactory as far as the general behavior of the curves is concerned, whereas the peak positions are not predicted correctly. Although the positions of the peaks could have been improved somewhat with a slightly different choice of Fermi-surface parameters, we did not attempt to do so because they also depend on the skin-depth phase. Furthermore, the departure of the orbits from circularity, which is of the order of $5 \%$, may have a considerable influence. ${ }^{10}$

For $\overrightarrow{\mathrm{B}}$ along the [011] direction, the cylinder model is inapplicable because of the rapid variation of $m_{c}$ with $k_{x}$, the momentum component parallel to $\overrightarrow{\mathrm{B}} \cdot{ }^{4,6}$ In this case, the dominant contributions are from those values of $k_{x}$ which ren- 
der stationary the -hases of the contributions from type-I and type-II trajectories. Using the calculated Fermi-surface data, ${ }^{4,6}$ we can show that for the type-II orbits there is no such stationary-phase point on the Fermi surface. Thus the type-II trajectories, which dominated the [100] spectrum, do not contribute to the [011] spectrum because of destructive interference. The type-I orbits, on the other hand, do offer a stationary-phase point which, according to the calculations, should be located at $k_{x} \approx 0.555$ $\times 10^{10} \mathrm{~m}^{-1}$ with a corresponding $k_{\perp}$ of $1.06 \times 10^{10}$ $\mathrm{m}^{-1}$ and $m_{c} / m_{0}=0.88$. Although the stationaryphase point is shifted towards smaller $k_{x}$ as $B$ approaches $B_{\text {S.E., its location is almost constant }}$ in the interval $100 \mathrm{G}<B<300 \mathrm{G}$ where most of the oscillations occur.

The calculated curve in Fig. 2 again represents a zero-parameter fit with $k_{x}$ assumed constant. In the range $B \leqslant 300 \mathrm{G}$ the agreement with the experimental tracing is quite good. As $B$ approaches $B_{\text {S.E. }}$ the stationary-phase point starts moving, and at the same time the sensitivity of the spectrum to the various parameters increases rapid1y. These features probably account for the discrepancy in the higher-field part of the spectrum. It should also be mentioned that the type-II orbits tend to develop a constant phase point in the limit as $B$ approaches $B_{\text {S.E. }}$ This may cause the appearance of a type-II signal, although with considerably reduced amplitude. On the low-field side, the sudden disappearance of the oscillations at $B=130 \mathrm{G}$ is probably caused by the emergence of two additional stationary-phase points giving rise to interference with the primary type-I oscillations. The signal which emerges below $B$ $\approx 100 \mathrm{G}$ is believed to be a size effect associated with the effective points $A$ and $B$ on a dog's bone orbit [Fig. 3(b)].

The possibility of obtaining precise Fermi-surface information from an experiment like the one described seems precluded, even in the case of circular orbits, because, as indicated above, the phases of the different contributions, and thus the positions of the peaks, depend on the assumption of a skin depth and on its phase $\chi$. Information independent of $\chi$ can be obtained by comparing the positions of adjacent peaks. Such information involves both the cyclotron frequency and averages of components of the Fermi velocity over parts of the electronic orbits. If the skindepth phase if relatively independent of magnetic field, then one can obtain velocity information by introducing a controlled phase shift $\psi$ in the leak signal. The maxima in the interference signal occur then, not when the phase delay is $2 \pi n$, but rather when it is $2 \pi n+\psi$. One can then measure the logarithmic derivatives, with respect to $\psi$, of the magnetic field at adjacent maxima of the (undifferentiated) interference signal. This will directly yield differences of inverse Fermi velocities at points about the Fermi surface. The positions of these points, at which the velocities are determined, however, still depend somewhat on the phase of the skin depth. Thus, it is evident that this kind of Fermi-surface information can probably not be used to construct a Fermisurface model but may be of value in providing a test of existing models at otherwise inaccessible points on the Fermi surface. In general, more oscillations, and thus more information, occur for type-I trajectories. These will be favored when there is no stationary point for type-II trajectories, a condition which obtains when, as a function of $k_{x}, v_{\perp}$ varies very little compared to $\omega_{c}$, as in the [011] direction of Ag. If the relevant part of the Fermi surface contains a center of symmetry, as in the case in the [100] direction of $\mathrm{Ag}$, then a stationary point for both types of oscillations will always occur there, and the type-II will dominate.

A more detailed report of this work will be presented elsewhere. We wish to thank N. E. Christensen for providing us with a wealth of calculated Fermi-surface information on $\mathrm{Ag}$, and Professor K. Sarmark for his continued interest in this work. One of us (D.S.F.) would also like to thank Professor Særmark for his kind hos pitality at Physics Laboratory I, The Technical University of Denmark.

*Current address: Department of Physics, University of Maryland, College Park, Md. 20742.

${ }^{1}$ V. F. Gantmakher, Zh. Eksp. Teor. Fiz. $\underline{42}, 1416$ (1962), and $\underline{43}, 345$ (1962) [Sov. Phys. JETP $\underline{15}, 982$ (1962), and $\overline{16}, 247$ (1963)].

${ }^{2}$ D. M. Sparlin [Phys. Lett. 25A, 46 (1967)] suggested that Fermi velocities could be measured by detecting the small phase lag $(\ll 2 \pi)$ occuring in a radio-frequency transmission experiment at the first-order size effect.

${ }^{3}$ R. G. Chambers, Proc. Phys. Soc., London, Sect. A 65, 458 (1952).

${ }^{4}$ N. E. Christensen, thesis, Technical University of Denmark, 1969 (unpublished), and private communication.

${ }^{5} \mathrm{~J}$. F. Koch and A. F. Kip, in Proceedings of the Ninth International Conference on Low Temperature Physics, Columbus, Ohio, 1964, edited by J. G. Daunt et al. (Plenum, New York, 1965); D. A. Smith, Proc. 
Roy. Soc., London Ser. A 296, 476 (1967); H. D. Drew, to be published.

${ }^{6}$ N. E. Christensen, Phys. Status Solidi 31, 635 (1969).

${ }^{7}$ H. V. Bohm and V. J. Easterling, Phys. Rev. 128, 1026 (1962).

${ }^{8} \mathrm{~J}$. O. Henningsen, Physics Laboratory I, Technical University of Denmark, Internal Report No. 77, 1970 (to be published).

${ }^{9}$ G. E. H. Reuter and E. H. Sondheimer, Proc. Roy.
Soc., Ser. A 195, 336 (1948).

${ }^{10}$ V. S. Edel'man and M. S. Khaikin, Zh. Eksp. Teor. Fiz. 53, 142 (1967) [Sov. Phys. JETP 26, 99 (1968)], observed some structure in the transmission spectrum of Bi near the cyclotron resonance cutoff. They attributed this partly to a resonant size effect of electrons being specularly scattered at one surface. In our data, there is no evidence for specular scattering at magnetic fields higher than $50 \mathrm{G}$.

\title{
Giant Diamagnetism and Impurity Configurations in Alkali Metals*
}

\author{
J. A. Rigert and C. P. Flynn \\ Department of Physics and Materials Research Laboratory, University of Mlinois, Urbana, Ilinois 61801 \\ (Received 19 February 1971)

\begin{abstract}
An unusually large diamagnetism $\sim-130 \mathrm{cgs} / \mathrm{mole}$ of certain positive-valence solutes in alkali metals is reported and explained in terms of a novel impurity configurational structure. Recent work on impurity-induced spin-flip scattering is also explained.
\end{abstract}

The observed large diamagnetism of positivevalence solutes in noble-metal solvents ${ }^{1}$ has previously found no explanation in sign or order of magnitude. Kohn and Luming ${ }^{2}$ and Graham and March ${ }^{3}$ discuss impurity magnetism in metals. For a linearized impurity screen they find small paramagnetic susceptibilities rather than the observed large diamagnetism $\sim-100 \mathrm{cgs} / \mathrm{mole}$. The discrepancy has tentatively been ascribed to band-structure effects. ${ }^{2}$ In this Letter we present more structured results for the susceptibilities of impurities in alkali metals, and show how these results are related to a novel impurity structure. The observed ${ }^{4,5}$ conduction-electron spin-flip rates induced by spin-orbit coupling in the impurity cell also find a natural explanation.

Figure 1 shows the measured susceptibilities of $\mathrm{Au}, \mathrm{Hg}, \mathrm{Tl}, \mathrm{Pb}, \mathrm{Bi}, \mathrm{Cd}, \mathrm{In}, \mathrm{Sn}, \mathrm{Sb}$, and $\mathrm{Te}$ in liquid $\mathrm{Na}$ at $300^{\circ} \mathrm{C}$. We have also obtained similar results for impurities in liquid $\mathrm{K}$. An attempt was made to measure $\chi$ for I also but, unfortunately, NaI proved insoluble in liquid $\mathrm{Na}$. Note, however, that the value $\chi=-52 \times 10^{-6} \mathrm{cgs} /$ mole ${ }^{6}$ for $\mathrm{I}^{-}$in NaI fits smoothly into the present results for solutes in liquid $\mathrm{Na}$. The peak diamagnetism for impurities of valence 5 and 6 is much larger than atomic susceptibilities. ${ }^{7}$ Since core orbitals can hardly be affected, the valence diamagnetism is larger than that of free atoms by a factor up to 4 . Band-structure effects can be discounted for this free-electron liquid solvent.

Also shown in Fig. 1 are the scattering cross sections deduced by Asik, Ball, and Slichter ${ }^{4}$ and by Cornell and Slichter ${ }^{5}$ for spin flips induced by spin-orbit coupling in the impurity cell. Clearly, these effects are also strongly valence dependent and giant diamagnetism emerges when the spin-flip scattering disappears. We believe that this happens because the two phenomena are sensitive to high densities of $p$-like impurity orbitals at the band bottom and at the Fermi surface, respectively.

The one-electron atomic $p$ levels fall below the pure $\mathrm{Na}$ band bottom for higher valence solutes. This suggests that $p$-like impurity orbitals may emerge below the bottom of narrow conduction bands in a manner similar to the transition from virtual to bound $4 f$ levels of rare-earth impurities recently reported by Blodgett and Flynn. ${ }^{8}$

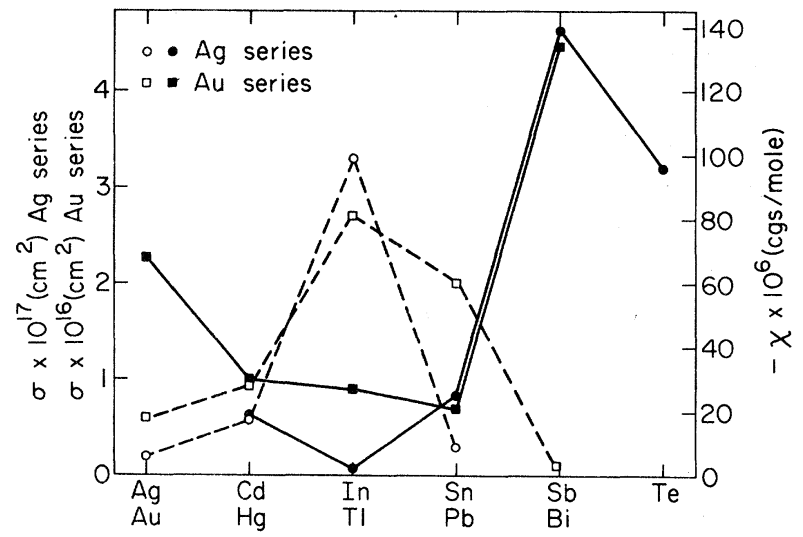

FIG. 1. The diamagnetic susceptibilities $\chi$ (closed points) and the spin-flip scattering cross sections $\sigma$ (open points) for impurities in $\mathrm{Na}$. 